\title{
Comparative Analysis of E-commerce Recommendation Strategies
}

\author{
Junhua Jiang ${ }^{1}$ \\ ${ }^{1}$ Changzhou Textile Garment Institute, Changzhou, Jiangsu, 213164 \\ hunter2011@foxmail.com
}

Keywords: E-commerce; Recommendation Strategies; Comparative Analysis

\begin{abstract}
This paper simply introduces some basic recommendation method used in E-commence website and mainly compares and analyzes the recommendation tactic used in China 2 E-commence websites: Amazon.com.cn and Douban.com.cn. By analyzing and comparing, this paper presents advantages and disadvantages existed in the china E-commence recommendation field.
\end{abstract}

\section{Introduction}

With the further expansion of the scale of e-commerce, our internet provides users with more choice, while its structure becomes more complex. On the one hand, users face a lot of product information helpless, users will often get lost in a lot of product information space, unable to successfully find the goods they need; on the other hand, businesses have lost contact with consumers. Recommended system simulation store merchandise sales staff to provide users with recommendations, to help users find the merchandise, so the successful completion of the purchase process, so users can effectively preserve and improve e-commerce sales system; businesses can also keep in touch with customers through recommendation system, rebuilding customer relationships.

On the one hand, e-commerce system needs to support the recommendation system to help users find desired products. On the other hand, e-commerce system's own characteristics but also conducive to the smooth implementation of the recommendation system, mainly including:

The wealth of data: all data collected rich e-commerce environment, such as user registration data, user transactions, user ratings data, user basket information, user browsing data. Rich data model for the establishment of a variety of recommendation, producing high quality recommendation possible. Electronic data collection: e-commerce environment in a variety of data collected electronically, reducing manual data collected human error that may occur, greatly reducing noise data, the credibility of the various data is relatively high, the data pretreatment is relatively simple. Easy to assess the effect of the recommendation: ROI implementation in e-commerce recommendation system is easy to access by increasing the amount of e-commerce Web sites, and e-commerce systems to increase sales and other indicators to assess directly.

Recommended system mainly through the following three ways to improve the ability to sell e-commerce system:

\section{The Recommendation Strategies}

Classified Browse. Browse the subject classification find based approach is in line with the characteristics of the user's habits, easy to be user acceptance disadvantage of this method is: a lot of content is difficult to determine their respective subclasses. Category Search method took more time, you must further reduced in accordance with the contents to find the target and taxonomy step to find the range, the lack of automation and intelligence. "many times users for their own needs is not clear, the system can not force the user one-time users to express all of their needs. Therefore, for these reasons, despite the classification lookup method is widely used, but there is actually a very serious problem, it must make up for its deficiencies by other methods.

Content-based Retrieval. Content-based retrieval is a traditional search technology, but also quite common and mature a technology widely used in various website search system and library departments in various fields. The main idea is to find the target based on the theme of keywords , 
is to find within the scope of the target keywords to find content that matches. this method advantage is that the method is relatively mature technology, users find easy to accept the result, the disadvantage is very dependent on the search results ${ }^{1}$ topic keywords determine ${ }^{\circ}$ difficult to find users new and potential points of interest.

The N Recommendations that Most Relevant Ones' Interest. Such methods also known as personalized recommendation, the main idea is to recommend the most user interest characteristic of $\mathrm{N}$ books, personalized recommendation degree maximum results. However, this method is currently used much at home and abroad, especially in China, almost no With this method, the main reason it is difficult to achieve, the main problem is difficult to achieve measure user interest in how, and how interest is calculated in line with the characteristics of the user's books recommended for this method, there are three types:. ${ }^{1}$ interest-based content recommendation : according to the user's browsing history (or purchase history, evaluation of history), to find the content that best matches the browsing history books, the advantage of this method is recommended by the results easy to understand, easy for the user to accept the disadvantage of recommendation difficult to achieve, especially for some bad description, it is difficult to characterize by several properties of objects, such as online bookstore does not reflect the delivery time, product quality assurance, and other aspects of quality of service issues; interest-based collaborative filtering recommendation: first find out the target user Interest most similar neighbor users, according to the evaluation results neighbors users or browsing history books predict the target user's interest. This approach content-based approach is radically different approach, it does not require a detailed description of books or the project, so the some bad characterizations of objects is particularly effective, but the method has many disadvantages, such as cold starting problems, sparse problem, algorithm robustness issues »Mixed Recommended: This method is a comprehensive and collaborative content recommendation recommended advantage of two methods, avoiding their disadvantages.

The Popular N Recommendations that Most Meet the Interest of Readers. This method is recommended to meet users' interests before the best-selling $\mathrm{N}$ present, and strive to improve the shortcomings of the former method of the lack of personalized, but inherits the advantages of the former method recommended by the results easy to understand. This approach is to pick out the most user interest features $\mathrm{N}$ book from best-selling books.

Letters Recommender. Its main approach is the book you want to find have certain aspects of the content, you can send e-mail to the system, the system offline find, and then another time to inform the search results to the user, so the advantage is that you can generally able to get their desired content, the disadvantage is because the system is offline, it will find a long time.

\section{The Feature Comparison of Domestic and Abroad B2C Websites}

Amazon.com is Personalized Recommendation Pioneer. Recommended combination Amazon recommendation system using collaborative filtering and content recommendation based on the recommended way to combine technology, weaknesses, effectively overcome the "cold start" problem, but also to ensure the recommended personalization and automation, you can also find new interest users .

Multidimensional Assessment multidimensional evaluation here means that the user can not only program evaluation, but also can make an assessment of the project reviews. For the system to learn the user's interests preferences, users need to be familiar with the commodity given level 1-5 evaluation, if the user is willing can also give a comment. The system is also set to comment on the contents of the evaluation mechanism, the user can leave a review scoring, scoring dimension is yes (helpful) or no (no help). So the system will automatically identify the most valuable user reviews, and priority is displayed. Multidimensional Assessment of setting allows the user to understand the product deeply, effectively help users decide whether to buy.

Social labels simply mean that record key project attributes. Users can create their own tags directory to find products with similar properties, which itself is a product recommendation. Users with similar interests and similar label products can establish contact, set up a virtual theme community. Users enjoy community exchange, but also spontaneously found his neighbor users, 
even if not a member of the community for the user, the system can also tag analyze the user's interest. Obviously, tag-based collaborative filtering user is very effective. Due to the properties of the product record tag in the project, for content-based recommendation will play a great help. This allows the user to identify, add self-organized activity established label effective platform for the socialization of the recommended way.

Personalized homepage registered users when they log into the system will be tailored Amazon.com for the user's personalized page. Including interest to the user's commodity recommendation New Products interest to the user, the user has to purchase goods related products, the user's browsing history and other information. On the personalized homepage, personalized recommendation occupy the first place, this layout allows users to take full note of the recommendation system function, to encourage users to use.

Douban is the Website that Recommended Good Things. Collection and evaluation of the Amazon also used the same watercress multidimensional evaluation, the user can make the evaluation of the project, you can also evaluate the usefulness of the content of user comments. In addition to the collection function also uses watercress. Users can be found in a book (audio / video) under "'" want to read "," reading "," read "label, select a label is equivalent to the book collection. Users of the collection project must be interest to the system will be based on project characteristics have been favorites to analyze the user's preference, thereby producing personalized recommendations. more involved in the evaluation and collection would "recommend" play a positive role.

Users can add tags socialization in the collection tag for the project, on the one hand to facilitate the user to organize their own collections catalog, on the other hand the user can find similar content items based on the tag. For example, in the content of the page item will show "watercress members used tags' Click the tab you'll see the list of projects based on the theme of many of the label. Users have the purpose of finding the required label, to find items of interest from the label list, similar to retrieve the directory, but the directory tag is added by the user spontaneously. Moreover, the system can also use the label property features items produced content-based recommendation. In short this allows the user to identify, add self-organized activities for socialization label recommended way to build an effective platform.

Neighbors and community neighbors and community are very unique place of Douban, recommended between users create the conditions. Watercress encourage users to find like-minded people with their own, add more neighbors and more will participate in the panel to help users discover new things of interest. Recommended neighbor often more innovative than the system more personalized items.

Active is recommended that each user is recommended contributors. Whether it is a book video, or comment content, and or discuss activities, as long as users feel good things can add to their recommendation among the "recommended" tag, so people with similar interests and his own are likely from the "My recommended "to find content of interest. Users can also make beans column, which is making a recommended list topic, your beans column more high ranking, the more likely to help more people.

The Compare of Douban and Amazon. Amazon's first personalized recommendation technology incorporated "personalized recommendation pioneer" to e-commerce sites, and in ten years time operation, the continuous research, improve the recommendation system. Data accumulated more than 10 years and continue to improve the achievements of the powerful Amazon now recommend strength. Systems based products and related accumulated huge user database, recording information including what each user has done in the last few years or a few minutes. For collaborative filtering recommendation system, there is nothing better than a powerful database has more advantage of it? In addition to the advantages of the database, Amazon intimate details of the design but also to recommend the system more perfect. In the Amazon website always reflect a concept: to help users to recommend a choice.

The first is a community watercress network, not in the trading relationship for the purpose, but to share the reading experience and get acquainted with the book club for the purpose of the 
community. Members of the watercress is a common interest to establish contact through audio books, watercress group is to establish contacts through joint participation of members. Then range of friends become more and more wide, the system make a group of people to meet another group of people and increase user mobility than simply search team. A big advantage is the watercress network of social relations and the people truly moved online, and formed a common interest for the media connections network. Personalized recommendation system algorithm is not limited to produce, but also a breakthrough will neighbor's recommendation applied to the system. Imitate real life word of mouth, spread neighbor's recommendation on the network. The Panel recommended that the collection and beans columns recommendation among users fully excavated. Compared with the recommended system algorithms, from people with similar interests get recommendations tend to be more effective, but there is no lack of interest in novel recommendation, you can also explore the new interest of the user. Watercress unearthed "bean column", "neighbor recommended" and "Favorite Group" is a very good recommendation application.

\section{Conclusion}

Internet development has greatly promoted the development of e-commerce, e-commerce development is an urgent need to support appropriate technology, our less developed recommendation technology hindering the development of e-commerce is the intention of this article, this paper comparative analyzes several recommendation technologies of typical B2C websites, points out the advantages and disadvantages between the $2 \mathrm{~B} 2 \mathrm{C}$ websites in terms of personalization and automation recommendation.

\section{References}

[1] Hendrik Drachsler, Hans G. K. Hummel and Rob Koper. Recommendations for Learners are Different Applying Memory-Based Recommender System Techniques to Life long learning. Proceedings of The 1st Workshop on Social Information Retrieval for Technology Enhanced Learning \& Exchange. 2000.

[2] Longworth N. Lifelong Learning in Action - Transforming Education in The 21stcentury, Rout ledge. 2003: 145-149.

[3] Badrul Sarwar, George Karypis, Joseph Konstan, and John Riedl. Item-Based Collaborative Filtering Recommendation Algorithms. Proceedings of The 10thinternational Conference On World Wide Web.2001.

[4] Linden, G. Smith, B. York, J. Amazon.Com Recommendations: Item-to-Item Collaborative Filtering:IEEE Internet Computing,2003,7(1). 